\title{
BMJ Open International phase I study protocol to develop a patient-reported outcome measure for adolescents and adults receiving gender-affirming treatments (the GENDER-Q)
}

To cite: Klassen AF, Kaur M, Johnson $\mathrm{N}$, et al. International phase I study protocol to develop a patient-reported outcome measure for adolescents and adults receiving gender-affirming treatments (the GENDER-Q). BMJ Open 2018;8:e25435. doi:10.1136/ bmjopen-2018-025435

- Prepublication history for this paper is available online. To view these files, please visit the journal online (http://dx.doi. org/10.1136/bmjopen-2018025435).

Received 16 July 2018 Revised 3 August 2018 Accepted 18 August 2018

Check for updates

(C) Author(s) (or their employer(s)) 2018. Re-use permitted under CC BY-NC. No commercial re-use. See rights and permissions. Published by BMJ.

For numbered affiliations see end of article.

Correspondence to Professor Anne F Klassen; aklass@mcmaster.ca

\section{ABSTRACT}

Introduction A critical barrier to outcome assessment in gender-affirming healthcare is the lack of a specific patient-reported outcome measure (PROM). This phase I protocol describes an international collaboration between investigators in Canada, Denmark, the Netherlands and the USA who have coalesced to develop a new PROM (ie, the GENDER-Q) to evaluate outcomes of psychological, hormonal and surgical gender-affirming treatments. Methods and analysis This phase I study uses an interpretive description approach. Participants aged 16 years and older seeking any form of gender-affirming treatments in centres located in Canada, Denmark, the Netherlands and the USA will be invited to take part in qualitative interviews. Participants will review BREAST-Q and FACE-Q scales hypothesised to contain content relevant to specific gender-affirming treatments. Interviews will elicit new concepts for additional scale development. Each interview will be digitally recorded, transcribed and coded. The main outcome of this phase I study will be the development of a conceptual framework and set of scales to measure outcomes important to evaluating gender-affirming treatments. To this end, analysis will be used to add/drop/revise items of existing scales to achieve content validity. For new concepts, coding will assign top-level domains and themes/ subthemes to participant quotes. Codes will be used to develop an item pool to inform scale development. Draft scales will be shown to transgender and gender diverse persons and experts to obtain feedback that will be used to refine and finalise the scales. The field-test version of the GENDER- $Q$ will be translated by following rigorous methods to prepare for the international field-test study. Ethics and dissemination This study is coordinated at McMaster University (Canada). Ethics board approval was received from the Hamilton Integrated Ethics Board (Canada), the Medical Ethical Committee at VUmc (The Netherlands) and Advarra (USA). Findings will be published in peer-reviewed journals and presented at national and international conferences and meetings.

\section{Strengths and limitations of this study}

- Recruitment of an international sample of participants will make it possible to identify concepts that are common to people seeking gender-affirming treatments who live in different countries and cultures

- The inclusion of adolescents and adults will enable us to identify concepts that are common across age.

- To enhance rigour, we adhere to published guidelines for determining content validity of existing scales, development of new scales and translation/ cultural adaptation of patient-reported outcome measures in other languages.

- We use a modern psychometric approach to ensure GENDER-Q scales are clinically meaningful and scientifically sound.

\section{INTRODUCTION}

The World Professional Association for Transgender Health (WPATH) is an international, multidisciplinary association that promotes evidence-based care, education, research, advocacy and public policy in transgender and gender diverse healthcare. ${ }^{1}$ As WPATH policies set the standard for clinical decision-making in the care of gender diverse individuals, the development of patient-centred outcome tools that measure issues that matter to persons from their perspectives was called for by the WPATH board in an open letter dated May 2018. ${ }^{2}$

Gender-affirming treatments are multifaceted and can directly impact appearance and how individuals function and feel. Such treatments have been shown to alleviate gender dysphoria and allow people who are 
transgender or gender diverse to live more comfortably with their bodies. ${ }^{3-5}$ Globally, a rapidly increasing number of individuals are seeking gender-affirming care. Concurrent with increasing demand, treatments are becoming more individualised as the concept of gender is increasingly recognised as existing along a continuum rather than binary. ${ }^{67}$ These changes make access to tools to enable shared decision-making and the assessment of care increasingly important. ${ }^{8}$

A current barrier to outcome assessment in gender-affirming healthcare is the lack of a specific patient-reported outcome measure (PROM) designed to evaluate treatment outcomes from the patient perspective. This barrier was highlighted in two recent systematic reviews, both of which identified that existing PROMs used in transgender surgery research are inadequate and called for new measures to be developed. ${ }^{9}{ }^{10}$ The development of a specific PROM is crucial because how someone feels and functions before and after gender-affirming treatments are concepts best assessed by self-report. ${ }^{11}$ Furthermore, outcomes of treatments to alleviate feelings of gender incongruence are not measured by generic PROMs, including the 36-Item Short Form Health Survey, ${ }^{12}$ which have been used to study gender-affirming surgery. Assessing outcomes that matter to patients, such as improvements in sexual well-being and body image after gender-affirming treatment, ${ }^{13-15}$ is essential to understanding the impact and success of treatments.

Specific scales are needed to cover the full range of gender-affirming treatments. Our team recently developed two scales to measure the appearance of the chest and nipples as a supplement to the BODY-Q. ${ }^{16}$ These scales were field-tested in an international (Canada, the USA, Denmark, the Netherlands) sample of 739 participants with different chest indications, including gynaecomastia, weight loss and gender-affirming chest surgery. ${ }^{17}$ To develop these scales, we used a modern psychometric approach to create clinically meaningful and scientifically sound PROM scales with high content validity ${ }^{18}{ }^{19}$ We followed international best practice guidelines that outline the latest methods for PROM development and validation, including guidelines for establishing content validity and special considerations for younger persons. ${ }^{1120-23}$ We followed a three-phased mixed methods approach that we previously published ${ }^{24}$ and is reproduced in figure 1. This three-phased approach ensures that a new PROM fulfils minimum standards regarding reliability and validity as described by the International Society for Quality of Life Research ${ }^{25}$ and the Consensus-based Standards for the Selection of Health Status Measurement Instruments. ${ }^{26}{ }^{27}$ Briefly, in phase I, using qualitative methods, a pool of items was generated. The item pool was developed into scales that were shown to patients and experts for feedback. This step ensured that the content of the new scales comprehensively measured outcomes that matter to patients and that instructions, response options and items were clear, meaningful and unambiguous. In phase II, the scales were field-tested in a large international sample. The items in each scale that represented the best indicators of outcome were identified and retained based on their performance against a set of psychometric criteria according to Rasch Measurement Theory (RMT) analysis. ${ }^{18}$ In this approach, the qualitative phase was crucial because the data were used to create, for each scale, a set of items that together mapped out a concept of interest on a clinical hierarchy. ${ }^{19}$ The RMT analysis was used to determine if the theorised concepts were supported by the field-test data (ie, fit of the data to the Rasch model). In phase III, patients are being invited to participate in a prospective study to measure change following gender-affirming chest surgery. Anchor-based and distribution-based methods will be used to examine responsiveness.

The following protocol describes an international collaboration between investigators in Canada, Denmark, the Netherlands and the USA who have coalesced to develop a new PROM for evaluating outcomes of gender-affirming treatments. The chest and nipples scales represent the first step in our programme of research to develop a comprehensive set of scales covering all types of gender-affirming treatments. Our overarching objective is to build on the chest and nipples scales to create a comprehensive PROM that is valid and reliable for use in research, quality improvement initiatives and in clinical care with individual patients seeking gender diverse healthcare treatments.

\section{METHODS AND ANALYSIS}

This protocol describes the phase I qualitative study to develop a set of independently functioning scales to evaluate gender-affirming treatments. We take an applied health services research approach called Interpretive Description. ${ }^{28}{ }^{29}$ This approach aims to generate knowledge relevant to the clinical context and presumes there is theoretical knowledge, clinical knowledge and a scientific basis informing a study.

We will conduct a series of interviews that will involve two parts. In part 1 , we will adapt existing BREAST- $Q^{30}$ and FACE- $Q^{31}{ }^{32}$ scales hypothesised to have relevant content by adding/dropping/revising items as needed. In part 2, we will develop scales for new concepts elicited. We previously used this two-part approach to establish content validity for existing scales and develop new scales for the FACE-Q Module for Children, and Young Adults described elsewhere. ${ }^{33}$

To establish content validity of existing scales, we follow guidance from the USA Food and Drug Administration (FDA), ${ }^{11}$ which recommends that new qualitative work, similar to that conducted when developing a PROM, can provide evidence of content validity for existing tools if patient interviews or focus groups are conducted using open-ended methods to elicit participant input. Our team will use cognitive interviews to identify relevant content within BREAST-Q and FACE- $Q$ scales and open-ended interviews to identify new concepts for scale development. 


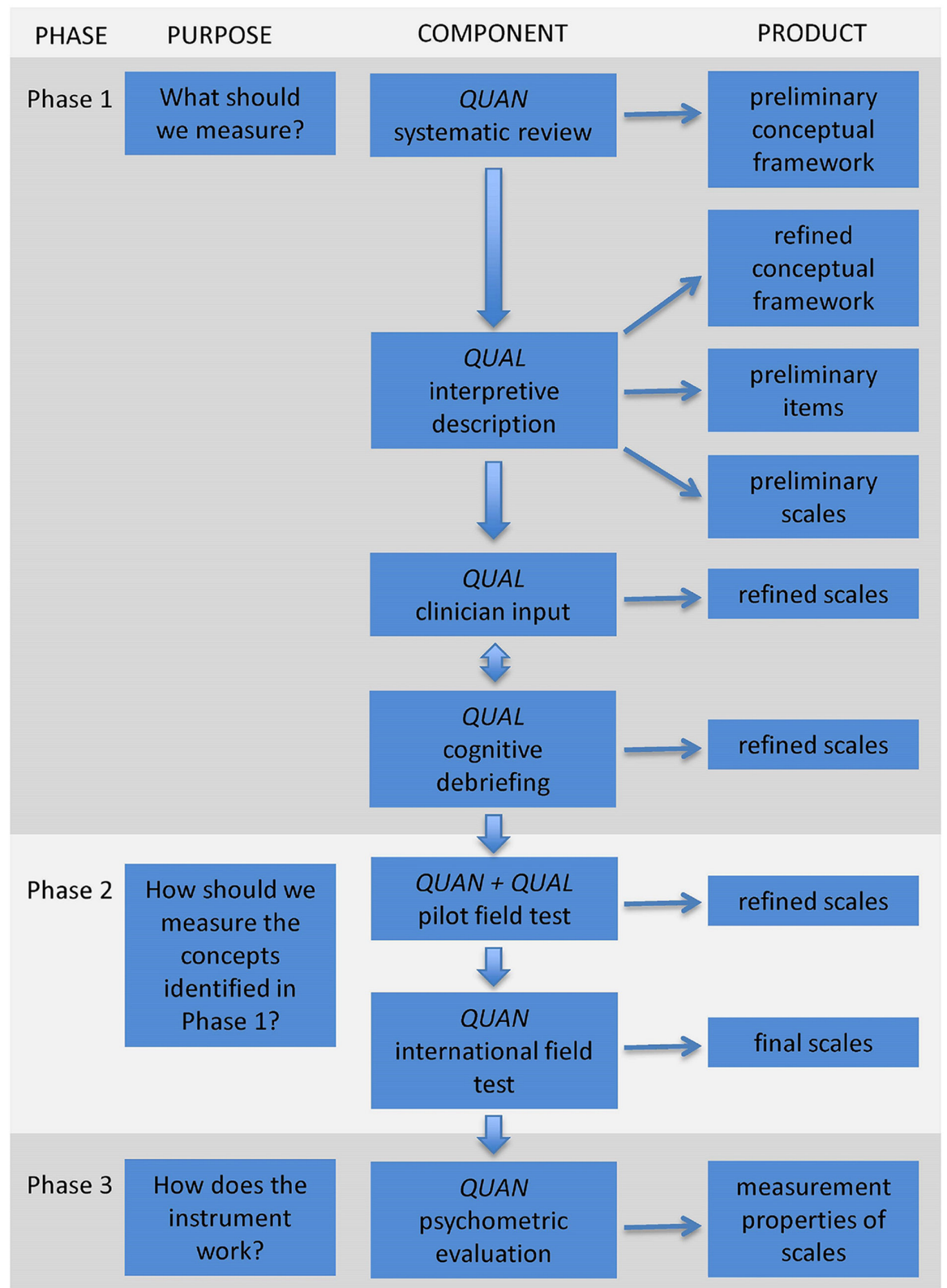

Figure 1 Flow diagram showing the multiphase mixed methods protocol for developing the GENDER-Q. Reproduced with permission from Wong Riff et al. ${ }^{24}$ QUAN, quantitative.

\section{Sample}

Participants will be purposively sampled to include a maximum variety of age, gender and treatment history (psychological, hormonal or surgical). Participants will be recruited by someone within the circle of care from each participating site or through local support groups. Contact details for those who express an interest in the study will be passed on to a member of the research team to schedule interviews. Participants will be recruited from the interdisciplinary clinic for transgender youth at McMaster Children's Hospital (Canada), the McLean Clinic (Canada), Women College Hospital (Canada), Aalborg University Hospital (Denmark), VU University Medical Center (Netherlands), Brownstein \& Crane Surgical Services (USA) and Align Surgical Associates (USA). We do not anticipate problems recruiting the necessary participants given the transgender focus and high volume of patients at these sites, as well as the expressed need for such a measure by the population themselves. Interviews will be conducted face-to-face or by phone depending on participant preference and logistics for travel.

\section{Part 1: cognitive interviews}

Sample size requirements for cognitive interviews are variable, with the number of interviews a function of the complexity of the PROM and the diversity of the population. ${ }^{34}$ For the review of existing BREAST-Q and FACE-Q scales, the FDA document advises the following for adapting an existing PROM for a new patient population: 'The sample size depends on the completeness of the information obtained from analysis of the transcripts. 
Generally, the number of patients is not as critical as interview quality and patient diversity included in the sample in relation to intended clinical trial population characteristics'. ${ }^{11}$ We will conduct as many cognitive interviews as are necessary to establish content validity for existing scales. Cognitive interviews will use the 'think aloud' method with probing to obtain feedback on all aspects of each scale. ${ }^{35}{ }^{36}$ Cognitive interviews are valuable for identifying missing content as well as tailoring existing item wording, item format and presentation to ensure respondents optimally understand them.

\section{Breasts}

Hormonal treatment can lead to breast development for some transgender and gender diverse women, ${ }^{37}$ but breast augmentation is often needed. The BREAST-Q is a PROM with separate modules for different types of breast surgery, including augmentation. The BREAST-Q was developed using interview data from 48 women, refined through focus groups and interviews involving 88 women, and field-tested with 908 presurgery and 1807 postsurgery women from Canada and the USA. ${ }^{30}$ The BREAST-Q has close to 50 translations and is used worldwide in clinical practice, quality improvement/benchmarking initiatives $^{38}{ }^{39}$ and research. ${ }^{40}$ The BREAST-Q has been used in research with transgender women ${ }^{41}{ }^{42}$ even though its development did not include any transgender or gender diverse individuals. We will identify relevant content within the BREAST-Q augmentation module to provide a means to measure satisfaction with breasts for transgender and gender diverse women. We hypothesise that some BREAST-Q items will be retained, some will need to be modified or dropped, and new items or scales may be needed.

\section{Face}

Male and female faces differ in obvious ways, especially the shape of the forehead, hairline, nose, lips, cheeks, chin, jawline, neck and facial hair. For transgender women, facial hair removal and facial feminisation surgery provide a means to achieve an appearance that is better aligned with gender identity. ${ }^{43}$ The FACE-Q aesthetic module ${ }^{3132}$ has 23 scales that measure the appearance of the face and specific facial areas. These scales were developed from 50 interviews with patients who were pre-treatment or post-treatment for surgical and minimally invasive aesthetic treatments and input from 26 experts. Scales were refined through 35 cognitive interviews and fieldtested in a sample of 988 cosmetic patients from Canada, the USA and the UK. Development of FACE-Q did not include people who are transgender or gender diverse. To provide scales that can be used to evaluate the effect of gender-affirming treatments on facial appearance, we will adapt the FACE-Q scales that measure face overall and features that differ the most between males/females, including the cheeks, chin, forehead, hairline, jawline, lips, neck and nose. Cognitive interviews will be used to determine whether the scales have content validity for people who are transgender or gender diverse. We hypothesise that in each scale, some items will be retained, some will need to be modified or dropped and new items or scales will be needed.

\section{Part 2: concept elicitation}

Scale review will be immediately followed by a qualitative interview to elicit new concepts not covered by the BREAST-Q and FACE-Q. An interview guide (see box 1) will be used to guide the interviews. Topics included in the guide were informed by PROMs reviewed in two recent systematic reviews of outcomes following gender-affirming treatments, ${ }^{910}$ and other phase I plastic surgery-specific PROM development studies performed by our team. The qualitative interviewer will dynamically adapt the interview as needed (eg, for younger participants) to avoid asking irrelevant questions or questions that might be too personal for some to discuss (eg, sexual well-being). As interviews progress, the interview guide will be iteratively adapted to include new concepts identified. Sampling and recruitment will continue until the point of saturation is reached, that is, no further new concepts are elicited from additional interviews. ${ }^{44}$

\section{Data analysis}

Both parts of the interviews will be digitally recorded and transcribed verbatim. Interviews performed in Denmark and the Netherlands will be translated into English prior to coding. Data collection and analysis will take place concurrently to build on the knowledge gained from each interview. Transcripts will be coded using a line-byline approach by one member of the research team and confirmed by a second member. Codes and data will be pasted into an Excel spreadsheet for analysis. For existing scales, feedback on the instructions, response options and items will be sorted by the scale/item number and examined to establish content validity for the existing scales by making necessary modifications. To develop new scales, patient quotes (participant words/phrases) from the semi-structured interviews that pertained to any aspect of outcome will be categorised into top-level domains and themes/subthemes. Qualitative analysis will lead to the refinement of a conceptual framework covering the concepts that matter to people seeking gender-affirming treatments. This framework will be used to guide scale development.

The coded material will be used to develop a comprehensive item pool within Excel. Items will retain participant-specific wording to ensure they are easy to understand and resonate with patients. As much as possible, positive or neutral wording will be adopted for items to minimise any negative impact of completing scales. The item pool will be analysed and used to develop a comprehensive set of independently functioning scales covering key aspects of the conceptual framework. Each scale will be assigned instructions and the most appropriate time frame and set of response options. 


\section{Box 1 Semi-structured interview guide}

\section{Part 1: review of BREAST-Q and FACE-Q scales Instructions}

- What are the instructions asking you to do? Please explain in your own words.

Is the time frame for responding to the scale appropriate?

- Are there any words we should change to make the instructions easier?

\section{Items}

In your own words, what is this item is asking?

What do you think of when answering this item?

- Are any words difficult to understand or offensive?

- Was this item hard or easy to answer and why?

Does this item measure an important issue for you?

What do you think about the response choices?

At the end of each scale

- In your own words, what is this group of items asking about?

- Does this group of items measure an important issue for you?

- Are there any items that do not belong with the rest?

Thinking about that group of items, what are we missing?

At the end of the interview

- What are your overall thoughts about the questionnaire?

- Is there anything we forgot to ask that is important to patients seeking treatment?

- Is there anything we should change about our questionnaire?

- Is there anything else that you would like to add or comment on?

\section{Part 2: concept elicitation for new scales}

\section{Experience of care}

- Can you tell me about the first time you asked for professional help with your gender identity?

- Can you tell me about the events that led to your decision to seek treatment?

- Have you experienced any barriers to obtaining gender-affirming treatments?

What are the people like who have cared for you? Probe: helpful, friendly, expertise, skill.

- What kind of information and advice were you given by healthcare providers? Probe: amount, content, interactions.

- How could healthcare professionals improve the quality of care provided to you?

\section{Treatments and recovery}

- What kinds of gender-affirming treatments have you had so far? Probe: psychological, hormonal, surgical.

What was the recovery like from surgical treatments? Probe: symptoms and impact on activities and daily life.

- What was good/bad about each gender-affirming treatment you have had?

- How happy/satisfied are you with each gender-affirming treatment you have had?

Do you plan to have any gender-affirming treatments in the future? If yes, which ones?

Appearance

- How important is your appearance to you?

- To what extent is your appearance aligned with your gender identity?

How would you describe your appearance? Probe: face/body/hair/ other.

- What do you like/dislike about the appearance? Probe: face/body/ hair/other.
Box 1 Continued

- Has your appearance changed with any gender-affirming treatments and how? Probe: face/body/hair/other.

- Is there anything else about your appearance that you would like to change? Probe: face/body/hair/other.

Voice

How important is how your voice sounds to you?

To what extent is your voice aligned with your gender identity?

- How would you describe your voice?

-What do you like/dislike about your voice?

- How has your voice changed with any gender-affirming treatments and how? Probe: do people respond to you differently?

Psychological

- How do you generally feel? Probe: negative (anxiety, depression, irritation) and positive (happy, post-traumatic growth).

- Does distress interfere with daily activities and how? Probe: work, social, dating.

- Has your emotional health changed with any gender-affirming treatments and how?

- Which gender-affirming treatments have helped the most in terms of emotional well-being?

Body image

- How do you feel about your face/body/hair/other? Probe: negative (self-conscious, uncomfortable, unattractive, abnormal) and positive (confident, attractive, normal).

- Do you hide or cover parts of your body or face or modify your voice to blend in? Probe for how and why.

- To what extent is your body image in line with your gender identity?

- Has your body image changed with any gender-affirming treatments and how?

- Which gender-affirming treatments have helped the most in terms of body image?

Social

What has it been like for you socially? Probe: school, work, friends, family, dating.

- Can you describe the kinds of emotional, informational and instrumental support you receive from your social network?

- Have you experienced any bullying, teasing, abuse, stigmatisation or discrimination? Probe: school, work, friends, family, dating and coping strategies.

- Are there things you want to do in life but do not because of gender-specific issues?

- Has your social life changed with any gender-affirming treatments and how? Probe: school, work, friends, family, dating.

- Which gender-affirming treatments have helped the most in terms of your social life?

Physical

- To what extent is your physical function aligned with your gender identity? Probe: mobility, strength, activities.

- How has your physical function changed with the gender-affirming treatments? Probe: mobility, strength, activities.

- Which gender-affirming treatments have helped the most in terms of physical function?

Sexual

How important is your sexual life and well-being to you?

- How do you feel about your body sexually? Probe: positive (confident, attractive, normal) and negatives (self-conscious, uncomfortable, unattractive, abnormal) and clothed/unclothed.

- Can you tell me about any problems or concerns with the sexual life related to your gender identity? 


\section{Box 1 Continued}

In what ways has gender-affirming treatment changed your genitalia? Probe: appearance, function.

- Has your sexual life changed with any gender-affirming treatment and how? Probe: frequency, quality, drive, arousal.

- Which gender-affirming treatments have helped the most in terms of your sexual life?

Additional questions

- Can you describe any other concerns or issues you experienced that we have not already covered?

- Thinking back over this interview, what are the most/least important issues that we have talked about?

\section{Scale refinement}

Refinement of new scales will take place using the same approach as described for existing scales, with multiple rounds of cognitive interviews using the 'think aloud' technique ${ }^{35}{ }^{36}$ Participants who took part in the initial interviews will be invited to take part in scale refinement. These interviews will be digitally recorded, transcribed and analysed to identify problematic items, response options and instruments. Before the final round of scale review by participants, the GENDER-Q will be shown to experts in transgender and gender diverse healthcare for feedback. The goal is to ensure that we have not overlooked any important concepts. We will design a survey using Research Electronic Data Capture, a secure web-based data collection system. ${ }^{45}$ Experts from around the world identified from our professional circles will be invited by email to provide feedback on existing content and to suggest missing content. Any changes suggested by experts will be reviewed in a final round of participant interviews to ensure that the field-test version of the GENDER-Q is composed of a set of scales that are easy to understand and comprehensively measure outcomes that matter to transgender and gender diverse people.

\section{Translations}

In preparation for an international field-test, the GENDER-Q will be translated into languages needed by participating sites. We will follow the methodology that was recommended for translations of PROMs in the field of plastic surgery, which we already applied to other PROMs developed by members of our team (eg, BODY$\mathrm{Q}^{46}$ CLEFT- $\left.\mathrm{Q}^{47}\right)$. This approach is in accordance with the International Society for Pharmacoeconomics and Outcomes Research ${ }^{48}$ and the World Health Organization ${ }^{49}$ recommendations for linguistic validations. Briefly, the translation will follow a rigorous process that involves two independent forward and one backward translation, an expert panel meeting and a series of cognitive patient interviews. The goal is to obtain a culturally adapted and equivalent translation of the GENDER-Q that is easily understood in the target language.

\section{Subsequent phases}

We will apply for grant funding to conduct phase II and III studies. The phase II field-test study will involve recruitment of a large international sample of participants. We will use the data collected to refine and validate GENDER-Q scales using RMT analysis. ${ }^{18}$ Our goal will be to identify the subset of items for each scale that represent the best indicators of outcome resulting in shorter clinically meaningful and scientifically sound scales. Items for retention will be identified based on their performance against a set of psychometric tests that are described in detail elsewhere. ${ }^{19}$ Once developed and validated, we will conduct further psychometric research to determine each scales' ability to measure clinical change following gender-affirming treatments (phase III studies).

\section{Patient and public involvement}

Our patient-oriented approach engages transgender and gender diverse people and healthcare providers in all stages of our research as experts and research team members whose input is crucial to the design of the study and development of content for GENDER-Q scales. All participants in the initial qualitative interviews will be invited to continue to collaborate in our study by taking part in scale refinement interviews where they can provide feedback on our findings and help to refine the final set of scales. At the VUmc hospital, regular meetings for transgender individuals are held to update them on the outcomes of conducted research. For all participants, we will disseminate feedback in the form a newsletter with links and information about presentations and publications.

\section{ETHICS}

Each participant will provide written and oral consent before participating. Given that transgender and gender diverse people can experience gender dysphoria and report higher than average rates of depression and suicide ideation, ${ }^{5-54}$ it could prove upsetting to participants to talk about their experiences. As necessary, we will provide a list of support services within the city and put patients in touch with a healthcare provider at the recruiting site to obtain support. Patient data will be de-identified at the transcription phase. All data will be kept secure and confidential following institution rules for data storage.

\section{DISSEMINATION}

To ensure uptake, once the GENDER-Q is developed, it will be made available free of charge to all non-profit users. Our team will seek to raise awareness and promote its use among stakeholders including patients, researchers, healthcare practitioners, decision-makers and policy-makers. To this end, our dissemination initiatives will include face-to-face interactions as well as electronic and hard-copy media, all effective strategies for research uptake. ${ }^{55}$ We will seek out opportunities to 
deliver presentations at national and international meetings. We will publish findings for the GENDER-Q in high impact journals that we know to be valued and read by our target audiences. Finally, we will use social media (eg, Twitter, Instagram) to spread awareness of our work to our network of followers.

\section{Author affiliations}

${ }^{1}$ McMaster University, Hamilton, Ontario, Canada

${ }^{2}$ Department of Medical Psychology, Amsterdam Public Health research institute, Center of Expertise on Gender Dysphoria, VU University Medical Center, Amsterdam, The Netherlands

${ }^{3}$ McLean Clinic, Mississauga, Ontario, Canada

${ }^{4}$ Division of Plastic Surgery, Department of Surgery, University of Washington Medical Center, Seattle, Washington, USA

${ }^{5}$ Department of Plastic Reconstructive and Hand Surgery, Amsterdam Public Health Research Institute, Center of Expertise on Gender Dysphoria, VU University Medical Center, Amsterdam, The Netherlands

${ }^{6}$ Department of Plastic and Reconstructive Surgery, Odense University Hospital, Odense, Denmark

${ }^{7}$ Brownstein \& Crane Surgical Services, Greenbrae, California, USA

${ }^{8}$ Fleming College-Emeritus, Peterborough, Ontario, Canada

${ }^{9}$ Women's College Hospital, Toronto, Ontario, Canada

${ }^{10}$ Amsterdam University Medical Centers, Amsterdam, The Netherlands

${ }^{11}$ Brigham and Women's Hospital, Boston, Massachusetts, USA

Contributors AFK, MK, NJ, GME, SM, MGM, M0, LP, WR, TS, KS, JSe, JSo, ALP conceived and designed the study. AFK, MK, NJ, BPCK, GME, SM, MGM, M0, LP, WR, TS, KS, JSe, JSo, TCvdG, MvdM-R, DY-A, ALP revised the article critically for important intellectual content and have approved the final version to be published.

Funding Phase I of this study is funded by research grants received from the Canadian Institutes for Health Research (ClHR), the Plastic Surgery Foundation and ZonMw (project number 636330001). The authors have no financial interest to declare in relation to the content of this article. The Article Processing Charge was paid from a CIHR grant.

Competing interests None declared.

Patient consent Not required.

Ethics approval This study has been approved by the Hamilton Integrated Research Ethics Board (HiREB). Institutional review board approval has also been obtained in the Netherlands by the Medical Ethical Committee at VUmc, and in the USA by Advarra for Brownstein \& Crane Surgical Services and Align Surgical Associates.

Provenance and peer review Not commissioned; peer reviewed for ethical and funding approval prior to submission.

Open access This is an open access article distributed in accordance with the Creative Commons Attribution Non Commercial (CC BY-NC 4.0) license, which permits others to distribute, remix, adapt, build upon this work non-commercially, and license their derivative works on different terms, provided the original work is properly cited, appropriate credit is given, any changes made indicated, and the use is non-commercial. See: http://creativecommons.org/licenses/by-nc/4.0/.

\section{REFERENCES}

1. Coleman E, Bockting W, Botzer M, et al. Standards of care for the health of transsexual, transgender, and gender-nonconforming people, version 7. Int J Transgend 2012;13:165-232.

2. WPATH. WPATH public policies. https://www.wpath.org/policies.

3. Gijs L, Brewaeys A. Surgical treatment of gender dysphoria in adults and adolescents: recent developments, effectiveness, and challenges. Annu Rev Sex Res 2007;18:178-224.

4. Murad MH, Elamin MB, Garcia MZ, et al. Hormonal therapy and sex reassignment: a systematic review and meta-analysis of quality of life and psychosocial outcomes. Clin Endocrinol 2010;72:214-31.

5. Zucker KJ, Lawrence AA, Kreukels BPC. Gender dysphoria in adults. Annu Rev Clin Psychol 2016;12:217-47.

6. Zucker KJ. Epidemiology of gender dysphoria and transgender identity. Sex Health 2017;14:404-11.
7. Beek TF, Kreukels BP, Cohen-Kettenis PT, et al. Partial treatment requests and underlying motives of applicants for gender affirming interventions. J Sex Med 2015;12:2201-5.

8. van de Grift TC, Mullender MG, Bouman MB. Shared decision making in gender-affirming surgery. implications for research and standards of care. J Sex Med 2018;15:813-5.

9. Barone M, Cogliandro A, Di Stefano N, et al. A systematic review of patient-reported outcome measures following transsexual surgery. Aesthetic Plast Surg 2017;41:700-13.

10. Andréasson M, Georgas K, Elander A, et al. Patient-reported outcome measures used in gender confirmation surgery: a systematic review. Plast Reconstr Surg 2018;141:1026-39.

11. U.S Department of Health and Human Services Food and Drug Administration. Guidance for industry patient-reported outcome measures: use in medical product development to support labeling claims. http://www.fda.gov/downloads/Drugs/Guidances/ UCM193282.pdf.

12. Lindqvist EK, Sigurjonsson H, Möllermark C, et al. Quality of life improves early after gender reassignment surgery in transgender women. Eur J Plast Surg 2017:40:223-6.

13. Nikkelen SWC, Kreukels BPC. Sexual experiences in transgender people: the role of desire for gender-confirming interventions, psychological well-being, and body satisfaction. J Sex Marital Ther 2018;44:370-81.

14. van de Grift TC, Elaut E, Cerwenka SC, et al. Effects of medical interventions on gender dysphoria and body image: a follow-up study. Psychosom Med 2017;79:815-23.

15. Auer MK, Liedl A, Fuss J, et al. High impact of sleeping problems on quality of life in transgender individuals: A cross-sectional multicenter study. PLoS One 2017;12:e0171640.

16. Klassen AF, Cano SJ, Alderman A, et al. The BODY-Q: a patientreported outcome instrument for weight loss and body contouring treatments. Plast Reconstr Surg Glob Open 2016;4:e679.

17 Klassen AF, Kaur M, Poulsen L, et al. Development of the BODY-Q chest module evaluating outcomes following chest contouring surgery. Plast Reconstr Surg 2018. doi: 10.1097/ PRS.0000000000004978 [Epub ahead of print].

18. Rasch G. Studies in mathematical psychology: 1. Copenhagen: Danmarks pædagogiske Institut, 1960.

19. Hobart J, Cano S. Improving the evaluation of therapeutic interventions in multiple sclerosis: the role of new psychometric methods. Health Technol Assess 2009;13:1-177.

20. Aaronson N, Alonso J, Burnam A, et al. Assessing health status and quality-of-life instruments: attributes and review criteria. Qual Life Res 2002;11:193-205

21. Patrick DL, Burke LB, Gwaltney CJ, et al. Content validityestablishing and reporting the evidence in newly developed patient-reported outcomes (PRO) instruments for medical product evaluation: ISPOR PRO good research practices task force report: part 1-eliciting concepts for a new PRO instrument. Value Health 2011;14:967-77.

22. Patrick DL, Burke LB, Gwaltney CJ, et al. Content validityestablishing and reporting the evidence in newly developed patient-reported outcomes (PRO) instruments for medical product evaluation: ISPOR PRO Good Research Practices Task Force report: part 2-assessing respondent understanding. Value Health 2011:14:978-88

23. Matza LS, Patrick DL, Riley AW, et al. Pediatric patient-reported outcome instruments for research to support medical product labeling: report of the ISPOR PRO good research practices for the assessment of children and adolescents task force. Value Health 2013:16:461-79.

24. Wong Riff KW, Tsangaris E, Goodacre T, et al. International multiphase mixed methods study protocol to develop a crosscultural patient-reported outcome instrument for children and young adults with cleft lip and/or palate (CLEFT-Q). BMJ Open 2017:7:e015467.

25. Reeve BB, Wyrwich KW, Wu AW, et al. ISOQOL recommends minimum standards for patient-reported outcome measures used in patient-centered outcomes and comparative effectiveness research. Qual Life Res 2013;22:1889-905.

26. Mokkink LB, Terwee CB, Patrick DL, et al. The COSMIN checklist for assessing the methodological quality of studies on measurement properties of health status measurement instruments: an international Delphi study. Qual Life Res 2010;19:539-49.

27. Terwee CB, Prinsen CAC, Chiarotto A, et al. COSMIN methodology for evaluating the content validity of patient-reported outcome measures: a Delphi study. Qual Life Res 2018;27:1159-70.

28. Thorne S, Kirkham SR, MacDonald-Emes J. Interpretive description: a noncategorical qualitative alternative for developing nursing knowledge. Res Nurs Health 1997;20:169-77. 
29. Thorne SE. Interpretive description. Developing qualitative inquiry. Walnut Creek (CA: Left Coast Press, 2008.

30. Pusic AL, Klassen AF, Scott AM, et al. Development of a new patientreported outcome measure for breast surgery: the BREAST-Q. Plast Reconstr Surg 2009;124:345-53.

31. Klassen AF, Cano SJ, Scott A, et al. Measuring patient-reported outcomes in facial aesthetic patients: development of the FACE-Q. Facial Plast Surg 2010;26:303-9.

32. Pusic AL, Klassen AF, Scott AM, et al. Development and psychometric evaluation of the FACE-Q satisfaction with appearance scale: a new patient-reported outcome instrument for facial aesthetics patients. Clin Plast Surg 2013;40:249-60.

33. Longmire NM, Wong Riff KWY, O'Hara JL, et al. Development of a new module of the face-q for children and young adults with diverse conditions associated with visible and/or functional facial differences. Facial Plast Surg 2017;33:499-508.

34. Willis GB. Cognitive interviewing: A tool for improving questionnaire design. Thousand Oaks, CA: Sage Publications, 2005.

35. Collins D. Pretesting survey instruments: an overview of cognitive methods. Qual Life Res 2003;12:229-38.

36. Van Someren M, Barnard Y, Sandberg J. The think-aloud method. London: Academic Press, 1994.

37. de Blok CJM, Klaver M, Wiepjes CM, et al. Breast development in transwomen after 1 year of cross-sex hormone therapy: results of a prospective multicenter study. J Clin Endocrinol Metab 2018;103:532-8.

38. ICHOM. Breast cancer. http://www.ichom.org/medical-conditions/ breast-cancer/.

39. Jeevan R, Cromwell DA, Browne JP, et al. Findings of a national comparative audit of mastectomy and breast reconstruction surgery in England. J Plast Reconstr Aesthet Surg 2014;67:1333-44.

40. Cohen WA, Mundy LR, Ballard TN, et al. The BREAST-Q in surgical research: A review of the literature 2009-2015. J Plast Reconstr Aesthet Surg 2016;69:149-62.

41. Agarwal CA, Scheefer MF, Wright LN, et al. Quality of life improvement after chest wall masculinization in female-to-male transgender patients: A prospective study using the BREAST-Q and Body Uneasiness Test. J Plast Reconstr Aesthet Surg 2018;71:651-7.

42. Weigert R, Frison E, Sessiecq Q, et al. Patient satisfaction with breasts and psychosocial, sexual, and physical well-being after breast augmentation in male-to-female transsexuals. Plast Reconstr Surg 2013;132:1421-9.

43. Ainsworth TA, Spiegel JH. Quality of life of individuals with and without facial feminization surgery or gender reassignment surgery. Qual Life Res 2010;19:1019-24.

44. Sandelowski M, saturation T. Given LM, The sage encyclopedia of qualitative methods. Thousand Oaks (CA): Sage, 2008:875-6.

45. Harris PA, Taylor R, Thielke R, et al. Research electronic data capture (REDCap)-a metadata-driven methodology and workflow process for providing translational research informatics support. J Biomed Inform 2009;42:377-81.

46. Poulsen $\mathrm{L}$, Rose $\mathrm{M}$, Klassen $\mathrm{A}$, et al. Danish translation and linguistic validation of the BODY-Q: a description of the process. Eur J Plast Surg 2017;40:29-38.

47. Tsangaris $\mathrm{E}$, Riff $\mathrm{K}$, Vargas $\mathrm{F}$, et al. Translation and cultural adaptation of the CLEFT-Q for use in Colombia, Chile, and Spain. Health Qual Life Outcomes 2017;15:228.

48. Wild D, Grove A, Martin M, et al. Principles of good practice for the translation and cultural adaptation process for patient-reported outcomes (pro) measures: report of the ispor task force for translation and cultural adaptation. Value Health 2005;8:94-104.

49. WHO (World Health Organization). The process of translation and adaptation of instruments. http://www.who.int/substance abuse/ research_tools/translation/en/.

50. Winter S, Diamond M, Green J, et al. Transgender people: health at the margins of society. Lancet 2016;388:390-400.

51. Reisner SL, Poteat T, Keatley J, et al. Global health burden and needs of transgender populations: a review. Lancet 2016;388:412-36.

52. Clements-Nolle K, Marx R, Katz M. Attempted suicide among transgender persons: The influence of gender-based discrimination and victimization. $J$ Homosex 2006;51:53-69.

53. Bauer GR, Scheim Al, Pyne J, et al. Intervenable factors associated with suicide risk in transgender persons: a respondent driven sampling study in Ontario, Canada. BMC Public Health 2015;15:525.

54. Rhodes $A E$, Bethell J, Bondy SJ. Suicidality, depression, and mental health service use in Canada. Can J Psychiatry 2006;51:35-41.

55. Dobbins M, Hanna SE, Ciliska D, et al. A randomized controlled trial evaluating the impact of knowledge translation and exchange strategies. Implement Sci 2009;4:61. 